\title{
Circulating triacylglycerol and apoE levels in response to EPA and docosahexaenoic acid supplementation in adult human subjects
}

\author{
Richard Buckley, Bethan Shewring, Rufus Turner, Parveen Yaqoob and Anne M. Minihane* \\ Hugh Sinclair Unit of Human Nutrition, School of Food Biosciences, University of Reading, Reading, UK \\ (Received 19 August 2003 - Revised 17 November 2003 - Accepted 19 November 2003)
}

\begin{abstract}
High doses of $n$-3 PUFA found in fish oils can reduce the circulating concentration of triacylglycerol (TG), which may contribute to the positive impact of these fatty acids on the risk of CVD. The present study aimed to establish the differential impact of EPA and docosahexaenoic (DHA) on plasma lipids and apo in adults. Forty-two normolipidaemic adult subjects completed a double-blind placebo controlled parallel study, receiving an EPA-rich oil (4.8 g EPA/d), DHA-rich oil (4.9 g DHA/d) or olive oil as control, for a period of 4 weeks. No effects of treatment on total cholesterol, LDL-cholesterol or HDL-cholesterol were evident. There was a significant $22 \%$ reduction in TG level relative to the control value following the DHA treatment $(P=0.032)$, with the $15 \%$ decrease in the EPA group failing to reach significance $(P=0 \cdot 258)$. There were no significant inter-group differences in response to treatment for plasma apoA1, $-\mathrm{C} 3$ or $-\mathrm{E}$ levels, although a significant $15 \%$ within-group increase in apoE was evident in the EPA $(P=0.006)$ and DHA $(P=0.003)$ groups. In addition, a within-group decrease in the apoA1:HDL-cholesterol ratio was observed in the DHA group, suggesting a positive impact of DHA on HDL particle size. The DHA intervention resulted in a significant increase in the proportion of EPA $P=0.000$ and DHA $P=0.000$ in plasma phospholipids, whilst significant increases in EPA $P=0.000$ and docosapentaenoic acid $P=0.002$, but not DHA $P=0 \cdot 193$, were evident following EPA supplementation $(P<0 \cdot 05)$. Our present results indicate that DHA may be more efficacious than EPA in improving the plasma lipid profile.
\end{abstract}

Eicosapentaenoic acid: Docosahexaenoic acid: Fish oils: Triacylglycerol: apolipoprotein E

Circulating triacylglycerol (TG) levels in the fasting and postprandial states are associated with the severity and progression of atherosclerosis (Hodis, 1999) and are now recognised as independent risk markers for CHD (Hokanson \& Austin, 1996; Karpe, 1997). The hypotriacylglycerolaemic potential of $n-3$ PUFA has been repeatedly demonstrated (Grimsgaard et al. 1997; Harris, 1997; Minihane et al. 2000). In a meta-analysis of sixty-five studies, Harris et al. (1997) concluded that an average dose of $\mathrm{EPA}+$ docosahexaenoic acid (DHA) of $4 \mathrm{~g} / \mathrm{d}$ resulted in a reduction of at least $25 \%$ in fasting TG levels in both normolipidaemic and hypertriacylglycerolaemic subjects. A decreased hepatic TG output as VLDL, attributed to an increased hepatic fatty acid oxidation and a decreased rate of lipogenesis, are considered to be partly responsible (Jump \& Clarke, 1999; Price et al. 2000). EPA and/or DHA, present in fish oil, are thought to serve as ligands for nuclear receptors such as PPAR- $\alpha$ (Jump \& Clarke, 1999; Clarke, 2000; Price et al. 2000) and sterol regulatory element binding proteins (Jump \& Clarke, 1999; Xu et al. 1999), which modulate the expression of key genes in these metabolic processes. However, these two fatty acids have traditionally been supplemented together in fish oils, and studies examining the individual impact of the $n-3$ PUFA on TG metabolism in human subjects are limited. In a study by Mori et al. (2000), where the diets of mildly hypercholesterolaemic male subjects were supplemented with $4 \mathrm{~g}$ EPA or DHA/d for 6 weeks, there was a trend towards a greater reduction in fasting TG levels in the DHA group, although the intergroup differences failed to reach significance. The primary aim of the current study was to determine the extent of any differential effects of EPA and DHA on plasma lipid levels, in view of their potential as cardioprotective agents.

In addition to the availability of fatty acids for TG synthesis by the liver, the apo content of lipoproteins is also an important determinant of circulating TG levels. Plasma apoE concentration accounts for $20-40 \%$ of the variation in circulating concentrations of TG (Salah et al. 1997), and is involved in many stages of TG-rich lipoprotein metabolism (Mahley, 1988), including VLDL and chylomicron synthesis (Mahley, 1988; Huang et al. 1999), VLDL catabolism to LDL (Huang et al. 1999) and the receptormediated clearance of TG-rich lipoprotein remnants by the liver (Krul et al. 1985; Mahley, 1988; Mahley \& Ji, 1999). apoC3 affects circulating TG levels by acting as an inhibitor of lipoprotein lipase-mediated hydrolysis of chylomicrons and VLDL and hepatic uptake of the resulting remnants (Jong et al. 1999). Although apo are important mediators of TG metabolism, the effect of $n-3$ PUFA 
on circulating levels is largely unknown and this was investigated in the current study.

\section{Methods}

\section{Study subjects}

Forty-five healthy adult volunteers, aged 20-70 years old, were recruited from the university campus and the local community. Exclusion criteria for participation in the study were: diagnosed diabetes or fasting glucose $>6.8 \mathrm{mmol} / \mathrm{l}$; liver or other endocrine dysfunction; evidence of CVD, including angina; hypolipidaemic therapy or any other medication known to interfere with lipid metabolism; consumption of fatty acid supplements; consumption of more than one portion of oily fish per week; weight-reducing diet; BMI $<20$ or $>32 \mathrm{~kg} / \mathrm{m}^{2}$; blood pressure $>160 / 95 \mathrm{mmHg}$. Further biochemical exclusion criteria included fasting TG $>4.0 \mathrm{mmol} / \mathrm{l}$ and fasting total cholesterol $>8.0 \mathrm{mmol} / \mathrm{l}$. The University of Reading Ethics Committee approved the study protocol and each individual gave written consent before participating.

Individuals were assigned to one of three study groups using blocked randomisation, with the groups matched for numbers of men and women and randomised on the basis of age and fasting TG levels, with the groups compared before the start of supplementation to ensure that there were no significant inter-group differences in BMI.

\section{Study design}

The present study was a double-blind, placebo-controlled, parallel study with subjects consuming $9 \mathrm{~g}$ EPA-rich oil, DHA-rich oil or a control oil each day for 4 weeks. The oils were provided as $9 \times 1 \mathrm{~g}$ oil capsules (Ocean Nutrition, Bedford, Nova Scotia, Canada); volunteers were asked to take three capsules with each of the three main meals in the day. The EPA and DHA capsules provided $4.8 \mathrm{~g}$ $\mathrm{EPA} / \mathrm{d}$ and $4.9 \mathrm{~g} \mathrm{DHA} / \mathrm{d}$ respectively. It must be noted that due to the difficulty in achieving $100 \%$ purity when synthesising the enriched oils from fish oil sources, the EPA-rich oil contained $8 \mathrm{~g}$ DHA/ $100 \mathrm{~g}$ total fatty acids $(720 \mathrm{mg})$, whilst the DHA-rich oil contained $9 \mathrm{~g}$ DHA/ $100 \mathrm{~g}$ total fatty acids $(810 \mathrm{mg})$. The major fatty acid in the control oil was oleic acid $(72 \mathrm{~g} / 100 \mathrm{~g}$ total fatty acids). The compositions of the test oils are given in Table 1. All three oils contained $2-4 \mathrm{mg}$ mixed natural tocopherols/g oil. Both the EPA- and DHA-enriched oils were supplemented with $7 \mathrm{mg}$ mixed natural tocopherols per capsule to prevent oxidation.

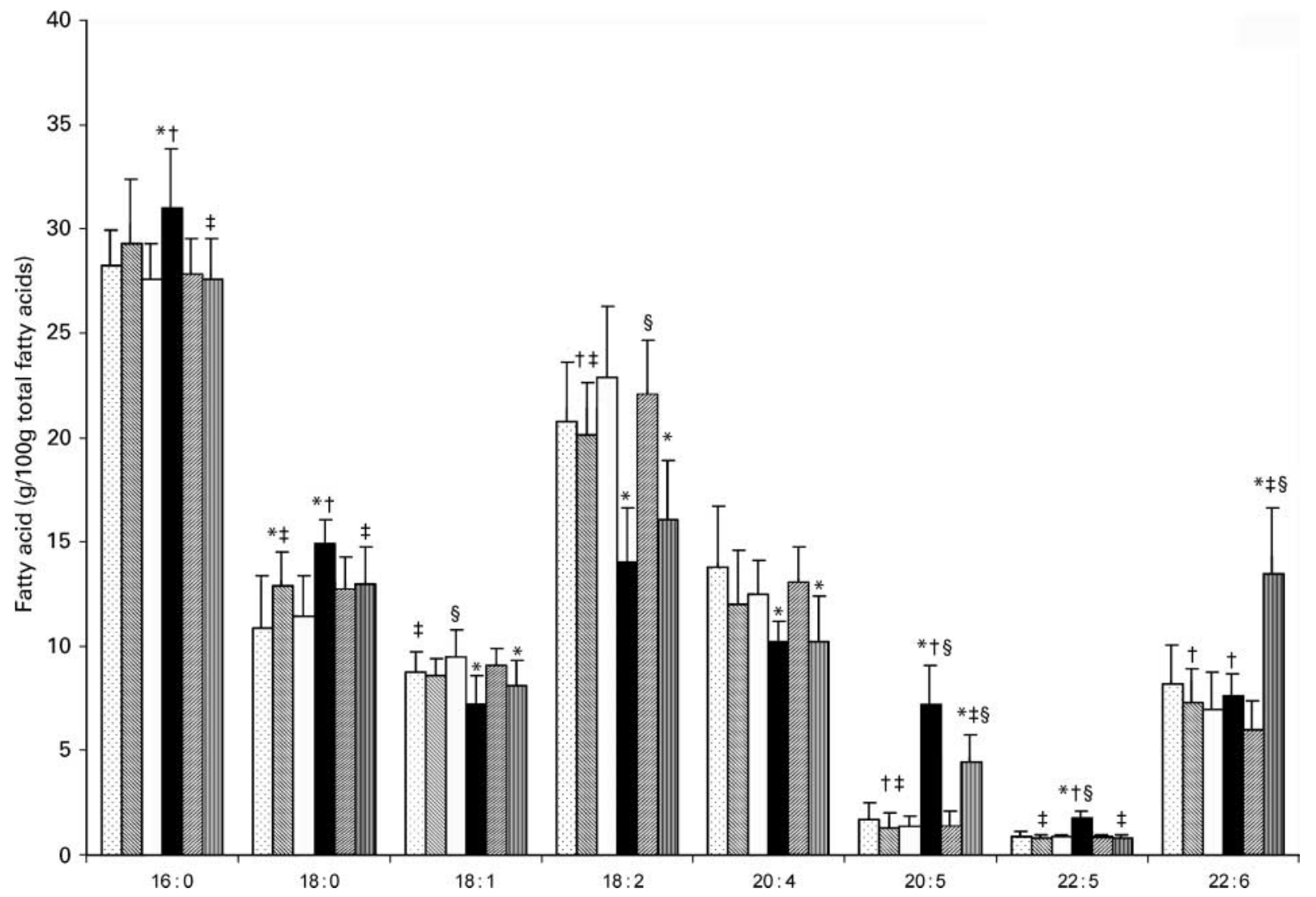

Fig. 1. The fatty acid composition of plasma phospholipids and baseline and post supplementation with EPA or docosahexaenoic acid (DHA).

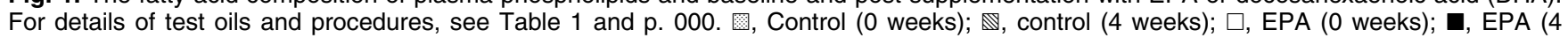
weeks); $\mathbb{2}$, DHA (0 weeks); 四, DHA (4 weeks). Intergroup differences and within-group differences were assessed using the Kruskal-Wallis and Wilcoxon signed-ranked tests respectively. Mean values were significantly different from those at baseline: ${ }^{\star} P<0.05$. Mean values were significantly different from those of DHA group at 4 weeks: $\dagger P<0.05$. Mean values were significantly different from those of EPA group at 4 weeks: $\ddagger P<0.05$. Mean values were significantly different from those of control group at 4 weeks: $§ P<0.05$. 
Table 1. The fatty acid composition of the study oils $(\mathrm{g} / 100 \mathrm{~g}$ total fatty acids)

\begin{tabular}{lrrr}
\hline & Control & EPA & DHA \\
\hline Palmitic acid $(16: 0)$ & $12 \cdot 1$ & 0.7 & 0.9 \\
Stearic acid $(18: 0)$ & $3 \cdot 2$ & $5 \cdot 0$ & 0.4 \\
Oleic acid $(18: 1)$ & $72 \cdot 0$ & $7 \cdot 4$ & 1.4 \\
Linoleic acid $(18: 2 n-6)$ & $10 \cdot 2$ & $1 \cdot 2$ & 0.4 \\
$\alpha$-Linolenic acid $(18: 3 n-3)$ & 0.5 & 0.4 & 0.4 \\
Arachidonic acid $(20: 4 n-6)$ & 0.0 & $2 \cdot 7$ & 0.6 \\
EPA $(20: 5 n-3)$ & 0.0 & $52 \cdot 8$ & 9.4 \\
Docosapentaenoic acid $(22: 5 n-3)$ & 0.0 & 1.8 & $10 \cdot 7$ \\
DHA $(22: 6 n-3)$ & 0.0 & $8 \cdot 1$ & 54.6 \\
\hline
\end{tabular}

DHA, docosahexaenoic acid.

Fasting blood samples $(20 \mathrm{ml})$ were collected at 0 and 4 weeks for analysis of plasma lipids, apo and phospholipid fatty acid compositions. Compliance was monitored using capsule count and by analysis of the phospholipid fatty acid profile.

\section{Biochemical analysis}

Fasting venous blood samples were collected into $10 \mathrm{ml}$ EDTA tubes and the samples centrifuged at $1600 \mathrm{~g}$ for $10 \mathrm{~min}$ at room temperature within $1 \mathrm{~h}$ of extracting the sample. Sub-samples of plasma were stored at $-80^{\circ} \mathrm{C}$ and analysed within 6 months of storage.

Analyses for total cholesterol, HDL-cholesterol, TG, apoE, apoC3 and apoA1 were conducted using the IL600 autoanalyser (Instrumentation Laboratories Ltd, Warrington, Ches., UK) and enzymic colorimetric kits (Instrumentation Laboratories Ltd). LDL-cholesterol levels were calculated using the Friedewald formula (Friedewald \& Levy, 1972).

Lipids were extracted from plasma samples with chloroform-methanol $(2: 1, \mathrm{v} / \mathrm{v})$ according to the method of Folch et al. (1957) and the phospholipids isolated from crude lipid using TLC with hexane-diethyl ether-acetic acid (90:30:1, by vol.) as the elution phase. Fatty acid methyl esters were prepared by incubation with $140 \mathrm{~g}$ boron trifluoride/l methanol at $80^{\circ} \mathrm{C}$ for $60 \mathrm{~min}$. Fatty acid methyl esters were re-extracted into hexane and analysed in a GC (model 6890; Hewlett Packard, Avondale, PA, USA) fitted with a $50 \mathrm{~m} \times 0.25 \mathrm{~mm}$ Chrompack 6173 fused silica capillary column with a film thickness of $0.25 \mu \mathrm{m}$. The carrier gas was $\mathrm{He}$ at $2.4 \mathrm{ml} / \mathrm{min}$ and a split-splitless injector was used with a 15:1 split-splitless ratio. Injector and detector temperatures were $240^{\circ} \mathrm{C}$ and $250^{\circ} \mathrm{C}$ respectively. The oven temperature was programmed to increase to $240^{\circ} \mathrm{C}$ in increments of $4^{\circ} \mathrm{C} / \mathrm{min}$ and then remain at $240^{\circ} \mathrm{C}$ for $4 \mathrm{~min}$. The separation was recorded with HP Chemstation software (Hewlett Packard). Fatty acid methyl esters were identified by comparison with standards run previously.

\section{Statistical analysis}

Group mean values are expressed as mean values with their standard errors. The data were checked for normality and skewed data was log transformed before statistical analysis. The significance of any differences between the groups at baseline or the impact of treatment on the percentage changes in each treatment group was determined using one-way ANOVA with post hoc comparisons of the lipid and apo variables carried out using the Tukey HSD post hoc test. Within treatment group, changes from 0-4 weeks were determined using paired Student's $t$ tests or Wilcoxon signed-rank tests (non-parametric data). A $P$ value $<0.05$ was considered significant. All statistical analysis was performed on the SPSS statistical package (version 11; SPSS Inc., Chicago, IL, USA).

\section{Results}

Forty-two of the forty-five volunteers completed the study. The mean age, numbers of men and women, BMI, and lipid and apo profiles of the group ( $n$ 42) overall and in the three treatment groups is given in Table 2 . The lipid profile of the present study population was representative of a typical UK adult population with mean total cholesterol, HDL-cholesterol and TG of 5.30 (SEM 0.15), 1.55 (SEM 0.06) and 1.17 (SEM 0.09) $\mathrm{mmol} / \mathrm{l}$ respectively. No significant inter-group differences were evident at baseline for any of the outcomes measured (Table 2). The capsules were well tolerated with an overall compliance (as determined by capsule count) of 94 (SEM 1) \% observed, with no significant difference between the treatment groups.

\section{Plasma phospholipid fatty acid composition}

No significant differences were observed in the fatty acid composition of the plasma phospholipids in any of the treatment groups at baseline. However, significant effects $(P=0.000)$ of treatment on post-supplementation levels of all fatty acids measured was observed, with the exception of arachidonic acid (Fig. 1). Relative to the control, intervention EPA supplementation resulted in significant increases in stearic acid, EPA and docosapentaenoic acid, and decreases in linoleic acid and oleic acid content $(P<0 \cdot 05)$. DHA intervention resulted in significant increases in the EPA and DHA content relative to the placebo intervention $(P<0 \cdot 05)$.

An examination of within-group changes in long-chain $n-3$ fatty acids in the intervention groups demonstrated significant increases in phospholipid EPA and docosapentaenoic acid in the EPA group (4.1- and 2.0-fold respectively, $P<0.05)$, with no significant effect on DHA. Significant 2.0- and 1.3-fold changes in EPA and DHA content were evident following DHA intervention $(P<0.05)$, with no significant effect on docosapentaenoic acid levels. The control treatment had no significant effect on phospholipid long-chain $n$-3 PUFA levels.

\section{Blood lipids and apo}

The individual treatments had no significant effect on circulating total cholesterol, LDL-cholesterol or HDL-cholesterol levels (Table 3). A significant effect of treatment was evident for TG levels $(P=0.038)$, with post hoc analysis demonstrating the $22 \%$ difference between the control and DHA groups to be significant $(P=0.032)$; the $15 \%$ 

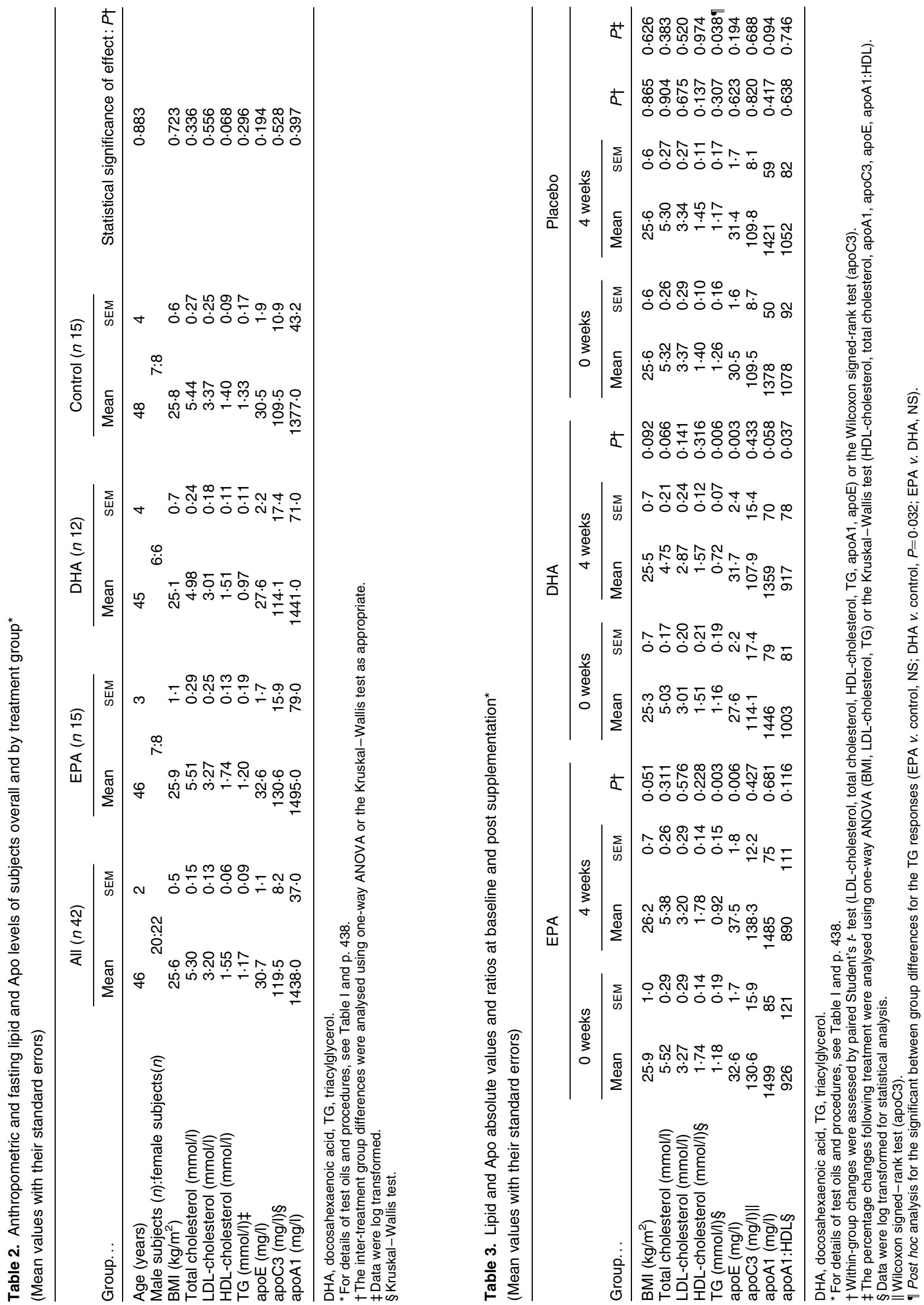
reduction in the EPA group relative to the control intervention failed to reach significance. No significant differences between the percentage changes in the EPA and DHA groups were observed. Significant within-group reductions in TG of $22 \%(P=0.001)$ and $38 \%(P=0.014)$ were evident following 4 weeks of EPA and DHA intervention respectively (paired $t$ tests).

No overall effects of treatment on circulating apoE, -C3 and -A1 levels were observed (Table 3). However, significant within-group increases in apoE levels of $15 \%$ were evident in the EPA $(P=0.006)$ and DHA $(P=0.003)$ treatment groups. Although no significant differences in apoA1 levels between treatment groups were evident, a significant within-group decrease in the apoA1:HDL ratio of $9 \%$ was observed following DHA treatment $(P=0 \cdot 037)$.

\section{Discussion}

Plasma TG are thought to affect atherogenesis directly and indirectly. In addition to the ability of small TG-rich lipoproteins to infiltrate and sequester lipid into the developing atheroma (Mamo et al. 1998; Gianturco \& Bradley, 1999), TG-rich lipoproteins are thought to be the principle metabolic determinant of the combined dyslipidaemia, referred to as the atherogenic lipoprotein phenotype; this condition is characterised by elevated fasting and postprandial TG, low HDL-cholesterol levels and a predominance of the dense putatively atherogenic LDL-3 particle ( $>40 \%$ total LDL) (Austin et al. 1988; Griffin et al. 1994). The atherogenic lipoprotein phenotype dyslipidaemia is commonly associated with obesity and diabetes and is thought to increase CHD risk by approximately 3-fold (Austin et al. 1988; Krauss, 1995).

Fish oils are considered the most effective dietary means of reducing TG levels, with intakes of $1.5 \mathrm{~g}$ EPA + DHA/d thought to be effective (Harris, 1997). However, concern exists regarding the impact of long-term consumption of these highly unsaturated fatty acids on overall LDL-cholesterol levels and the oxidisability of the LDL particle (Minihane et al. 2000; Leigh-Firbank et al. 2002); identification of the active and efficacious $n-3$ PUFA in fish oil could allow its selective consumption as a hypotriacylglycerolaemic agent.

The expected changes in post-supplementation plasma phospholipids were observed with EPA intervention, resulting in significant increases in both EPA and docosapentaenoic acid, but no effect on DHA. In contrast, the DHA intervention resulted in significant increases in both EPA and DHA, suggesting retroconversion of the longer 22:6 fatty acid (DHA) to its 20:5 derivative (EPA). These findings are consistent with previously reported findings (Grimsgaard et al. 1997; Mori et al. 2000).

In the present study, the DHA intervention was found to be more effective than EPA in reducing TG levels. Relative to the olive-oil placebo group, only the $22 \%$ reduction $(P=0.032)$ in the DHA group remained significant, with a non-significant $15 \%$ difference between the EPA and olive-oil group. However, it should be noted that the DHA oil contained a greater amount of docosapentaenoic acid relative to the EPA treatment. Although it is not known if docosapentaenoic acid has any TG-lowering potential, it is worthy of further investigation.

A number of studies have examined the individual impact of DHA on lipid levels in human subjects. Intakes of $1 \cdot 2-6 \cdot 0 \mathrm{~g} \mathrm{DHA} / \mathrm{d}$ for $6-15$ weeks resulted in a $17-26 \%$ reduction in plasma TG levels, demonstrating the potential of DHA to lower TG (Agren et al. 1996; Davidson et al. 1997; Nelson et al. 1997). Only a limited number of studies have directly compared the two fish oil fatty acids. In a group of mildly hypertriacylglycerolaemic younger adults (mean age 30 years), Rambjør et al. (1996) observed greater reductions in TG following EPA compared with DHA intervention. However, conclusions were drawn based on the combined data from three individual studies, which varied in their design (both crossover and parallel) and the form of administration of the fatty acids (liquid and encapsulated forms). In two more recent intervention trials where $3.5-4.0 \mathrm{~g}$ EPA or DHA were fed for intervention periods of 6-7 weeks, consistently greater reductions in TG were observed following DHA intervention (Grimsgaard et al. 1997; Mori et al. 2000). However, in contrast to the current study, the inter-group differences failed to reach statistical significance. In a study examining the impact of EPA $v$. DHA on postprandial TG metabolism, a 19 and $49 \%$ reduction in postprandial lipaemia was evident following consumption of $4 \mathrm{~g}$ EPA or $4 \mathrm{~g}$ DHA respectively for 4 weeks, again suggesting DHA to be more potent (Hansen et al. 1998).

Although there is some recent evidence suggesting that $n$-3 PUFA induces increases in systemic lipoprotein lipase activity that may contribute to the hypotriacylglycerolaemic effect of fish oils (Khan et al. 2002; Park \& Harris 2003), the positive effect on circulating TG levels is considered to be predominantly attributable to the effects of EPA and DHA on the hepatic availability of fatty acids for VLDL synthesis (Jump \& Clarke, 1999; Price et al. 2000). Early work examining the differential effects of EPA and DHA in vitro indicated that both fatty acids had the capacity to inhibit hepatic TG synthesis and secretion (Madsen et al. 1999). Subsequent in vivo studies in rats suggested that EPA was the principal modulator of fatty acid oxidation and lipogenesis and that DHA had little effect on hepatic TG output (Zhang et al. 1991; Froyland et al. 1996; Willumsen et al. 1996). However, extrapolation of these results to man is difficult: rodents have a very different lipoprotein profile to human subjects and excessive doses of EPA and DHA (1-2 g/kg per d) were fed to bring about the observed changes.

Although apo are the integral protein component of lipoproteins and involved in the secretion of chylomicrons and VLDL and their subsequent systemic metabolism, clearance from the circulation, the impact of fish oil fatty acids on apo metabolism is relatively unknown. However, in a study conducted by Nordøy et al. (2001) in hyperlipidaemic patients, atorvastatin $(10 \mathrm{mg} / \mathrm{d}$ for 5 weeks) treatment resulted in highly significant reductions in circulating apoE levels, although supplementation with $\mathrm{EPA}+\mathrm{DHA}$ ( $2 \mathrm{~g} / \mathrm{d}$ for 5 weeks) resulted in no further reduction relative to the maize-oil fed control group. 
In the current study, a within-group increase of $15 \%$ in apoE levels was evident following both EPA and DHA intervention. apoE is synthesised mainly by the liver as a 299 amino acid protein; it is thought to be involved in VLDL assembly, either as a chaperone of nascent lipidpoor apoB from the rough endoplasmic reticulum to the smooth endoplasmic reticulum for further lipidation, or to be involved in the transfer of newly synthesised TG to the smooth endoplasmic reticulum (Hamilton et al. 1990; Huang et al. 1999). An increased hepatic expression of the apoE gene has been shown to be associated with increased hepatic VLDL-TG output (Huang et al. 1998). However, apoE is also an essential modulator of 30$50 \%$ of TG-rich lipoprotein clearance by the liver as it acts as a ligand for the hepatic LDL-receptor and the LDL-receptor-related protein (Krul et al. 1985; Tomiyasu et al. 2001). Therefore, there is a good deal of uncertainty in interpreting the physiological significance of modest increases in plasma apoE levels. Huang et al. (1999) examined the association between over expression of the human apoE gene and the kinetics of lipoprotein metabolism in transgenic rabbits. By increasing the concentrations of apoE from 0 to $>200 \mathrm{mg} / \mathrm{l}$ a net increase in circulating TG levels was observed. However, increases in the plasma levels of the apoE protein up to a concentration of $100 \mathrm{mg} / \mathrm{l}$ resulted in a net reduction in TG levels, attributable to a greater efficiency in TG-rich lipoprotein clearance relative to the negative effect of increased VLDL production. Therefore, in the current study the $15 \%$ increase in apoE concentration in the fish oil fatty acid groups from a basal level of approximately $30 \mathrm{mg} / \mathrm{l}$ may have had a positive effect on whole-body TG metabolism and contributed to the lower TG levels following EPA and DHA intervention.

Although no significant effect of treatment on overall apoA1 levels was evident, within-group decreases in apoA1:HDL-cholesterol in the EPA and DHA groups were observed, with the $9 \%$ decrease in the DHA group reaching significance $(P=0.037)$. This result is suggestive of an effect of supplementation on HDL particle size, with a shift towards the larger, more cholesterol-rich $\mathrm{HDL}_{2}$ particle. Increased HDL particle size is thought to serve as a marker for more efficient reverse cholesterol transport and reduced CHD risk (Alagona et al. 2002). The results are consistent with the observations of Mori et al. (2000) and Rambjør et al. (1996), who observed significant increases in $\mathrm{HDL}_{2}$ following DHA but not EPA supplementation.

In conclusion, the current study indicates that both EPA and DHA intervention have TG-lowering properties, with DHA being a more potent hypotriacylglycerolaemic agent. Further work is needed to confirm that $n-3$ PUFA increase circulating apoE levels and that such increases contribute to the TG reductions observed.

\section{Acknowledgements}

We are grateful to Ocean Nutrition, Bedford, Nova Scotia, Canada, for providing us with the study capsules, and to all the study participants.

\section{References}

Ågren JJ, Hänninen O, Julkunen A, Fogelholm L, Vidgren H, Schwab U, Pynnönen O \& Uusitupa M (1996) Fish diet, fish oil and docosahexaenoic acid rich oil lower fasting and postprandial plasma lipid levels. Eur J Clin Nutr 50, 765-771.

Alagona C, Soro A, Ylitalo K, Salonen JT \& Taskinen MR (2002) A low high density lipoprotein (HDL) level is associated with carotid artery intima-media thickness in asymptomatic members of low HDL families. Atherosclerosis 165, 309-316.

Austin MA, Breslow JL, Hennekens CH, Buring JE, Willett WC \& Krauss RM (1988) Low density lipoprotein subclass patterns and risk of myocardial infarction. J Am Med Assoc 260, 917-921.

Clarke SD (2000) Polyunsaturated fatty acid regulation of gene transcription: a mechanism to improve energy balance and insulin resistance. Br J Nutr 83, S59-S66.

Davidson MH, Maki KC, Kalkowski JA, Schaefer EJ, Torri SA \& Drennan KB (1997) Effects of docosahexaenoic acid on serum lipoproteins in patients with combined hyperlipidaemia: a randomized, double-blind placebo-controlled trial. J Am Coll Nutr 16, 236-243.

Folch J, Lees M \& Sloane Stanley GH (1957) A simple method for the isolation and purification of total lipides from animal studies. J Biol Chem 226, 497-509.

Friedewald WT \& Levy RI (1972) Estimation of the concentration of low-density lipoprotein cholesterol in plasma, without use of the preparative ultracentrifuge. Clin Chem 18, 499-502.

Gianturco SH \& Bradley WA (1999) Pathophysiology of triglyceride-rich lipoproteins in atherothrombosis: cellular aspects. Clin Cardiol 22, II7-II14.

Frøyland L, Vaagenes H, Asiedu DK, Garras A, Lie O \& Berge RK (1996) Chronic administration of eicosapentaenoic acid and docosahexaenoic acid as ethyl esters reduced plasma cholesterol and changed the fatty acid composition in rat blood and organs. Lipids 31, 169-178.

Griffin BA, Freeman DJ, Tait GW, Thomson J, Caslake MJ, Packard CJ \& Shepherd J (1994) Role of plasma triglyceride in the regulation of plasma low density lipoprotein (LDL) subfractions: relative contribution of small, dense LDL to coronary heart disease risk. Atherosclerosis 106, 241-253.

Grimsgaard S, Bonaa KH, Hansen JB \& Nordoy A (1997) Highly purified eicosapentaenoic acid and docosahexaenoic acid in humans have similar triacylglycerol-lowering effects but divergent effects on serum fatty acids. Am J Clin Nutr 66, 649-659.

Hamilton RL, Wong JS, Guo LS, Krisans S \& Havel RJ (1990) Apolipoprotein E localization in rat hepatocytes by immunogold labelling of cryothin sections. J Lipid Res 31, 1589-1603.

Hansen JB, Grimsgaard S, Nilsen H, Nordoy A \& Bonaa KH (1998) Effect of highly purified eicosapentaenoic acid and docosahexaenoic acid on fatty acid absorption, incorporation into serum phospholipids and postprandial triglyceridemia. Lipids 33, 31-38.

Harris WS (1997) $n-3$ Fatty acids and serum lipoproteins: human studies. Am J Clin Nutr 65, 1645S-1654S.

Hodis HN (1999) Triglyceride rich lipoprotein remnant particles and risk of atherosclerosis. Circulation 99, 2852-2854.

Hokanson JE \& Austin MA (1996) Plasma triglyceride is a risk factor for cardiovascular disease independent of high-density lipoprotein cholesterol; a meta analysis of population based studies. J Cardiovasc Risk 3, 213-219.

Huang Y, Ji Z-S, Brecht WJ, Rall SC, Taylor JM \& Mahley RW (1999) Overexpression of apolipoprotein E3 in transgenic rabbits causes combined hyperlipidemia by stimulating hepatic VLDL production and impairing VLDL lipolysis. Arterioscler Thromb Vasc Biol 19, 2952-2959. 
Huang Y, Liu XQ, Rall SC Jr, Taylor JM, von Eckardstein A, Assmann G \& Mahley RW (1998) Overexpression and accumulation of apolipoprotein $\mathrm{E}$ as a cause of hypertriglyceridaemia. J Biol Chem 273, 26388-26393.

Jong MC, Hofker MH \& Havekes LM (1999) Role of apoCs in lipoprotein metabolism. Arterioscler Thromb Vasc Biol 19, 472-498.

Jump DB \& Clarke SD (1999) Regulation of gene expression by dietary fat. Annu Rev Nutr 19, 63-90.

Karpe F (1997) Postprandial lipid metabolism in relation to coronary heart disease. Proc Nutr Soc 56, 671-678.

Khan S, Minihane AM, Talmud PJ, Wright JW, Murphy MC, Williams CM \& Griffin BA (2002) Dietary long chain $n-3$ PUFA increase LPL gene expression in adipose tissue of subjects with an atherogenic lipoprotein phenotype. J Lipid Res 43, 979-985.

Krauss RM (1995) Dense low-density lipoproteins and coronary artery disease. Am J Cardiol 75, 53B-57B.

Krul ES, Tikkanen MJ, Cole TG, Davie JM \& Schonfeld G (1985) Roles of apolipoproteins B and E in the cellular binding of very low-density lipoproteins. J Clin Invest 75, 361-369.

Leigh-Firbank EC, Minihane AM, Leake DS, Wright JW, Murphy MC, Briffin BA \& Williams CM (2002) Eicosapentaenoic acid and docosahexaenoic acid from fish oils: differential associations with lipid responses. Br J Nutr 87, 435-445.

Madsen L, Rustan AC, Vaagenes H, Berge K, Dyrøy E \& Berge RK (1999) Eicosapentaenoic and docosahexaenoic acid affect mitochondrial and peroxisomal fatty acid oxidation in relation to substrate preference. Lipids 34, 951-963.

Mahley RW (1988) apolipoprotein E: cholesterol transport protein with expanding role in cell biology. Science $\mathbf{2 4 0}$, $622-630$.

Mahley RW \& Ji Z-S (1999) Remnant lipoprotein metabolism: key pathways involving cell-surface heparin sulphate proteoglycans and apolipoprotein E. J Lipid Res 40, 1-16.

Mamo JCL, Proctor SD \& Smith D (1998) Retention of chylomicron remnants by arterial tissue: importance of an efficient clearance mechanism from plasma. Atherosclerosis 141, S63-S69.

Minihane AM, Khan S, Leigh-Firbank EC, Talmud PJ, Wright JW, Murphy MC, Griffin BA \& Williams CM (2000) apoE polymorphism and fish oil supplementation in subjects with an atherogenic lipoprotein phenotype (ALP). Arterioscler Thromb Vasc Biol 20, 1990-1997.

Mori TA, Burke V, Puddey IB, Watts GF, O'Neal DN, Best JD \& Beilin LJ (2000) Purified eicosapentaenoic and docosapentaenoic acids have differential effects on serum lipids and lipoproteins, LDL particle size, glucose, and insulin in mildly hyperlipidemic men. Am J Clin Nutr 71, 1085-1094.

Nelson GJ, Schmidt PC, Bartolini GL, Kelley DS \& Kyle D (1997) The effect of dietary docosahexaenoic acid on plasma lipoproteins and tissue fatty acid composition in humans. Lipids 32, 1137-1146.

Nordøy A, Hansen J-B, Brox J \& Svensson B (2001) Effects of atorvastatin and $\omega-3$ fatty acids on LDL subfractions and postprandial hyperlipidemia in patients with combined hyperlipidemia. Nutr Metab Cardiovasc Dis 11, 7-16.

Park Y \& Harris WS (2003) Omega-3 fatty acid supplementation accelerates chylomicron triglyceride clearance. J Lipid Res $\mathbf{4 4}$, 455-463.

Price PT, Nelson CM \& Clarke SD (2000) Omega-3 polyunsaturated fatty acid and regulation of gene expression. Curr Opin Lipidol 11, 3-7.

Rambjør GS, Wålen AI, Windsor SL \& Harris WS (1996) Eicosapentaenoic acid is primarily responsible for hypotriglyceridemic effect of fish oil in humans. Lipids 31, S45-S49.

Salah D, Bohnet K, Gueguen R, Siest G \& Visvikis S (1997) Combined effects of lipoprotein lipase and apolipoprotein $\mathrm{E}$ polymorphisms on lipid and lipoprotein levels in the Stanislas cohort. J Lipid Res 38, 904-912.

Tomiyasu K, Walsh BW, Ikewaki K, Judge H \& Sacks FM (2001) Differential metabolism of human Veldt according to content of apoE and apoC-III. Arterioscler Thromb Vasc Biol 21, $1494-1500$.

Willumsen N, Vaagenes H, Lie O, Rustan AC \& Berge RK (1996) Eicosapentaenoic acid but not docosahexaenoic acid increases mitochondrial fatty acid oxidation and upregulates 2,4-dienoyl-CoA reductase gene expression in rats. Lipids 31, 579-592.

Xu J, Nakamura MT, Cho HP \& Clarke SD (1999) Sterol regulatory element binding protein- 1 expression is suppressed by dietary polyunsaturated fatty acids. $J$ Biol Chem 274, 840-848.

Zhang ZJ, Wilcox HG, Elam MB, Castellani LW \& Heimberg M (1991) Metabolism of $n-3$ polyunsaturated fatty acids by the isolated perfused rat liver. Lipids 26, 504-511. 